\title{
Marie-Antoinette dans les fonds des archives nationales
}

\section{Christine Nougaret}

\section{(2) OpenEdition \\ 1 Journals}

\section{Édition électronique}

URL : https://journals.openedition.org/ahrf/1877

DOI : 10.4000/ahrf.1877

ISSN : 1952-403X

Éditeur :

Armand Colin, Société des études robespierristes

\section{Édition imprimée}

Date de publication : 1 décembre 2004

Pagination : 129-136

ISSN : 0003-4436

\section{Référence électronique}

Christine Nougaret, «Marie-Antoinette dans les fonds des archives nationales », Annales historiques de la Révolution française [En ligne], 338 | octobre-décembre 2004, mis en ligne le 22 mars 2006, consulté le 22 avril 2022. URL : http://journals.openedition.org/ahrf/1877 ; DOI : https://doi.org/10.4000/ahrf. 1877

Ce document a été généré automatiquement le 22 avril 2022.

Tous droits réservés 


\title{
Marie-Antoinette dans les fonds des archives nationales
}

\author{
Christine Nougaret
}

1 Pendant plus de vingt ans, Marie-Antoinette a été sur le devant de la scène historique française et depuis deux siècles elle ne cesse de susciter des études historiques ou des biographies romancées et d'intéresser, sinon de fasciner, par son destin exceptionnel ${ }^{1}$. Pour tous les auteurs de ces travaux, le recours aux Archives nationales est incontournable. Pourtant, à y regarder de plus près, il n'existe pas aux Archives nationales de fonds Marie-Antoinette, c'est-à-dire d'ensemble constitué et organisé par elle et conservé en l'état, mais seulement des archives relatives à la souveraine et de trop rares documents émanant d'elle.

2 Plusieurs raisons à cela. En premier lieu, Marie-Antoinette ne fut pas ou peu productrice d'archives. Comme le soulignait Stefan Zweig, « Marie-Antoinette ...n'était pas une grande épistolière» et elle n'écrivait que contrainte et forcée ${ }^{2}$. Selon Suzanne d'Huart, ancienne responsable de la Section des archives privées des Archives nationales, qui s'intéressa aux autographes de la souveraine, on a recensé tout au plus 400 lettres écrites par Marie-Antoinette pendant les 23 années passées en France 3 .

Outre de rares lettres adressées à la princesse de Polignac ou à la princesse de RohanGuéméné, on lui connaît quatre correspondances suivies : la première avec sa mère et ses frères empereurs d'Autriche, conservée aux Archives d'Etat à Vienne et publiée, en 1874, par Arneth et Geoffroy ${ }^{4}$; la deuxième avec la landgrave Louise de HesseDarmstadt, publiée dès 1865 par le comte de Reiset ${ }^{5}$; la troisième avec Axel de Fersen, éditée par le petit neveu de celui-ci le baron Klinckowström ${ }^{6}$ et dont les épaves sont conservées aux Archives nationales depuis 1982; enfin, la quatrième avec Barnave, publiée par Alma Söderhjelm ${ }^{7}$ et conservée en Suède. Peu épistolière, Marie-Antoinette n'était pas davantage écrivassière : elle n'a pas tenu de journal, par exemple, à l'inverse de Louis XVI.

3 En second lieu, les papiers de Marie-Antoinette, et en particulier la correspondance reçue par elle, ont subi plusieurs vagues de destructions. Nous savons ainsi, grâce aux mémoires de Mme Campan, première femme de chambre de la reine, que Marie- 
Antoinette a brûlé une grande quantité de ses papiers, au lendemain du 14 juillet 1789, quand elle a songé à quitter Versailles pour la frontière ; elle n'a gardé par-devers elle que ceux qu'elle souhaitait emporter et qui la suivirent finalement dans sa résidence forcée aux Tuileries à partir du 6 octobre $1789^{8}$. Un an et demi plus tard, le 20 juin 1791, la tentative de fuite de la famille royale échouait à Varennes. Dès le 21 juin, l'Assemblée constituante ordonnait la mise sous scellés des appartements des Tuileries et se saisissait des papiers trouvés chez les souverains ${ }^{9}$. Un décret du 25 juin en ordonnait le transfert aux Archives nationales. Parallèlement, le comité des recherches ordonnait la rétention de toute lettre adressée à la famille royale, à Fersen ou à toute personne attachée à la famille royale. De retour aux Tuileries et placés désormais sous stricte surveillance, la famille royale n'eut plus que le recours de la correspondance secrète pour communiquer avec l'extérieur. De cette période datent les lettres à Barnave et à Fersen précédemment citées.

4 Le durcissement de la Révolution, le report de l'aide des souverains étrangers, les menaces pesant sur la famille royale, la crainte d'une nouvelle invasion des Tuileries conduisirent les souverains à se défaire d'une partie de leurs papiers. Marie-Antoinette brûla une partie des siens et remit à Fersen, lors de leur dernière entrevue, en février 1792, plusieurs lettres qu'elle jugeait nécessaire de conserver et, en particulier, la correspondance reçue par elle de Barnave et de Fersen. L'abolition de la royauté le 10 août 1792 et l'internement consécutif de Louis XVI et Marie-Antoinette mirent un terme définitif à toute correspondance de leur part. Les Tuileries furent alors livrées au pillage et c'est l'intervention de la Législative qui permit dans l'automne suivant de récupérer les papiers volés en août aux Tuileries. Confiés à la garde des Archives nationales, ces papiers des souverains servirent de pièces à conviction dans les procès de Louis XVI et de Marie-Antoinette, ce qui leur vaut d'être aujourd'hui conservés dans le fonds du tribunal révolutionnaire ${ }^{10}$.

5 Au total, que nous reste-t-il comme archives de Marie-Antoinette? En réalité aucun papier personnel, la quasi-totalité de la correspondance reçue par la souveraine ayant disparu. L'essentiel des documents en notre possession consiste en les dossiers des administrations et tribunaux qui ont échappé aux triages mais non au morcellement dans le cadre de classement des Archives nationales.

6 Pour l'Ancien Régime, il s'agit tout d'abord des archives de la maison du roi et de celles de la maison de la reine qui comprennent les dossiers de gestion des biens de la couronne affectés aux souverains ${ }^{11}$. Y figurent en particulier les documents comptables ayant trait au fonctionnement de la maison de Marie-Antoinette et à ses dépenses aussi bien utilitaires que somptuaires. Les épaves des papiers de Campan, secrétaire du cabinet de Marie-Antoinette à partir de 1778, acquises par les Archives nationales en 1985 , complètent cet ensemble ${ }^{12}$. De la maison du roi procèdent aussi les dossiers sur le mariage, en 1770, du dauphin Louis et de l'archiduchesse Marie-Antoinette. En deuxième lieu, le Centre historique abrite les archives du bureau de ville de Paris qui ont conservé le souvenir de toutes les manifestations et festivités ayant accompagné la vie des souverains, à l'occasion des maladies et grossesses de Marie-Antoinette, des naissances et obsèques des enfants royaux, de ses visites et déplacements... ${ }^{13}$. Le troisième ensemble est constitué par les archives judiciaires relatives à l'affaire du collier de la reine, et en particulier les procédures contre les différents protagonistes dont le cardinal de Rohan et Cagliostro pour ne citer que ceux-ci ${ }^{14}$. S'y ajoutent les papiers séquestrés du cardinal de Rohan ${ }^{15}$. Les archives Castries, déposées aux Archives 
nationales en 1971, apportent un complément à ces dossiers d'archives publiques grâce au journal du maréchal de Castries, ministre de la Marine de Louis XVI, qui fut chargé avec Vergennes, ministre des Affaires étrangères, de l'instruction de l'affaire avant que celle-ci ne fut envoyée devant les tribunaux ${ }^{16}$.

7 Plus dramatiques, les archives de la période révolutionnaire relatives à MarieAntoinette concernent les dernières années de sa vie jusqu'à son exécution. Les archives des assemblées, composées pour l'essentiel d'actes officiels, rendent compte de la détérioration progressive de la situation des souverains de l'été 1789 jusqu'à leur déchéance en août 1792. Elles abritent, en particulier, les papiers saisis aux Tuileries, dans l'armoire de fer et dans l'appartement de la reine ${ }^{17}$. Dans les archives du pouvoir exécutif, celles du comité de sûreté générale ${ }^{18}$ comprennent les dossiers relatifs à la captivité de la famille royale au Temple, et celles du comité des recherches ${ }^{19}$ les dénonciations et calomnies contre la reine. Le fonds du tribunal révolutionnaire, enfin, conserve les dossiers relatifs à la détention de Marie-Antoinette à la Conciergerie, à la tentative d'évasion connue sous le nom de conspiration de l'œillet, au procès de la souveraine et à son exécution ${ }^{20}$. Les archives publiques officielles dominent donc très nettement. Néanmoins quelques écrits autographes de Marie-Antoinette se retrouvent aux Archives nationales.

8 Comme il a été dit en introduction, il existe peu d'écrits autographes de MarieAntoinette. Quantité de faux ont été fabriqués au milieu du XIXe siècle par le baron Feuillet de Conches, chef du protocole de Louis-Philippe et maître des cérémonies de Napoléon III $^{21}$. Les travaux d'expertise et d'érudition de la fin du XIXe siècle et du début $\mathrm{du}$ XXe ont permis de discerner le vrai d'avec le faux ${ }^{22}$. Sur les 400 lettres autographes authentiques aujourd'hui connues, 19 sont conservées au Centre historique des Archives nationales : 13 pour l'Ancien Régime et 6 pour la période 1791-1793. Les treize lettres de Marie-Antoinette à la princesse de Rohan-Guéméné, gouvernante des Enfants de France jusqu'à la fameuse faillite du prince de Rohan-Guéméné en 1782, figurent dans les archives Rohan-Bouillon, propriété de l'Etat depuis $1985^{23}$. Dans ces lettres, Marie-Antoinette donne ses consignes à la princesse pour l'éducation de Mme Royale et lui répercute le résultat de ses interventions auprès de Louis XVI. Toute autre est la tonalité des courriers rédigés pendant la Révolution. Marie-Antoinette, en résidence forcée aux Tuileries, passe désormais de longues heures à écrire pour solliciter l'appui à la contre-révolution des souverains étrangers. Toute correspondance lui étant interdite, elle apprend le chiffrage et le déchiffrement, le recours à l'encre sympathique et au citron et les envois secrets à Fersen sous de fausses identités. Mme Campan a relaté dans ses mémoires le côté fastidieux de ces travaux de copies et décryptage. Marie-Antoinette elle-même s'en est plainte dans une lettre à Fersen du 2 novembre 1791 (copie) : «Adieu, je suis fatiguée à force d'écritures, lui dit-elle; jamais je n'ai fait tel métier et je crains toujours d'oublier, ou de mettre quelques bêtises $»^{24}$. De cette période ne subsistent aux Archives nationales que 5 lettres autographes écrites entre septembre 1791 et janvier $1792: 1$ à son frère Léopold (8 septembre 1791) saisie à Bruxelles et confiée aux Archives nationales ${ }^{25}$ et 4 à Fersen, acquises par les Archives nationales en $1982^{26}$. A ce lot, il convient d'ajouter 23 lettres de Marie-Antoinette dont nous ne possédons que la transcription par Fersen ${ }^{27}$ : la destruction des originaux par son petit neveu le baron Klinckowström et le caviardage de certains passages ont nourri de nombreuses hypothèses sur les relations amoureuses de Marie-Antoinette et Fersen. La dernière lettre autographe de Marie-Antoinette conservée au Centre historique est aussi la plus célèbre puisqu'il s'agit de l'ultime, qu'elle a écrite à la 
Conciergerie le matin même de son exécution, le 16 octobre $1793^{28}$. Dans cette lettre fameuse, appelée parfois testament de Marie-Antoinette, et maintes fois reproduite, Marie-Antoinette recommande son âme à Dieu et ses enfants à Mme Elisabeth.

Bien que n'ayant pas laissé d'archives personnelles, Marie-Antoinette, de son mariage à sa mort, est bien présente dans les Archives nationales à travers des sources variées pour lesquelles l'intérêt des chercheurs ne faiblit pas. Fersen le premier l'avait prévu quand il relatait au duc Charles, régent de Suède, le 20 octobre 1793, " les derniers moments d'une reine que ses malheurs et son courage feront vivre à jamais dans la postérité ».

\section{NOTES}

1.Parmi ses biographes les plus récents on citera : Simone Bertière (Marie-Antoinette l'insoumise, Paris, éditions de Fallois, 2002), Evelyne Lever (Marie-Antoinette, Paris, Fayard, 1991), Chantal Thomas (La reine scélérate, Paris, Le Seuil, 1989) et chez les romanciers : Françoise Chandernagor (La chambre, Paris, Gallimard, 2002) ou encore Chantal Thomas (Les adieux à la reine, Paris, Le Seuil, 2002).

2.Stefan Zweig, Marie-Antoinette, Paris, 1937.

3.Suzanne d'Huart, «Vraies ou fausses ? Les lettres de Marie-Antoinette », dans L'Histoire, n 51, décembre 1982, pp. 91-93.

4.Chevalier Alfred d'Arneth et Auguste Geoffroy, Marie-Antoinette : correspondance secrète entre Marie-Thérèse et le comte de Mercy-Argenteau, avec les lettres de Marie-Thérèse et de Marie-Antoinette, Paris, Firmin-Didot Frères, fils et Cie, 1874, 3 vol.

5.Lettres de la reine Marie-Antoinette à la landgrave Louise de Hesse-Darmstadt, Paris, Plon, 1865.

6.Baron Rudolf Maurits de Klinckowstrom, Le comte de Fersen et la cour de France, extraits des papiers du grand maréchal de Suède, comte Jean-Axel de Fersen, publiés par son petit-neveu, Paris, 1877, 2 vol.

7.Alma Soderhjelm, Marie-Antoinette et Barnave, correspondance secrète (juillet 1791-janvier 1792), Paris, Armand Colin, 1934. [comprend les rapports d'expertise d'A. de Bouard de Laforest, G. Bourgin et R. Anchel].

8.Madame Campan, Mémoires sur la vie de Marie-Antoinette, reine de France et de Navarre, Paris, Firmin-Didot Frères, fils et Cie, 1867 (Bibliothèque des mémoires relatifs à l'histoire de France pendant le 18e siècle).

9.Archives nationales, série $C$.

10.Ibid., série W.

11.Ibid., séries $\mathrm{K}$ et 01.

12.Ibid., 440 AP 2.

13.Ibid., série $\mathrm{K}$.

14.Ibid., série X.

15.Ibid., série $T$.

16.Ibid., 306 AP 17.

17.Ibid., série $C$. 
18.Ibid., série F7.

19.Ibid., série D.

20.Ibid., série W.

21.Félix-Sébastien Feuillet de Conches, Marie-Antoinette et Madame Elisabeth, Paris, 1865.

22.Voir notes 1 et 2 ; voir aussi Maxime de La Rocheterie et marquis de Beaucourt,

Lettres de Marie-Antoinette, recueil des lettres authentiques de la reine, publié pour la Société d'histoire contemporaine, Paris, Picard, 1895-1896, ainsi que Georges Girard,

Correspondance entre Marie-Antoinette et Marie-Thérèse, Paris, 1933.

23.Archives nationales, 273 AP 8.

24.Ibid., 440 AP 1.

25.Ibid., AE II 1241.

26.Ibid., 440 AP 1.

27.Ibid.

28. Archives nationales, AE I 7-8 $n^{\circ} 3$. 